\title{
Segmentation of Textured Image Based on Gauss-MRF in Overcomplete Brushlet Domain
}

\author{
Liu Xuena \\ College of Information Science and Technology \\ Bohai University \\ Jinzhou Liaoning,China \\ Liu-xuena@163.com
}

\author{
Sun Decai \\ College of Information Science and Technology \\ Bohai University \\ Jinzhou Liaoning,China \\ sdecai@163.com
}

\begin{abstract}
The focus of this paper is the improvement of the quality of texture image segmentation. We proposed a new unsupervised segmentation method based on Overcomplete Brushlet transform and Gaussian Markov Random Field. A texture image was transformed to Overcomplete Brushlet domain, in order to extract its high dimensional singularity information. In view of the influences of the spectral information and the spatial correlations between pixels to the segmentation result, Markov Random Filed model is used in the process of both feature extraction and region segmentation: Gauss Markov model is used to evaluate the arguments of the feature field; the probability of the marker field is calculated through Gibbs distribution function based on the second order neighborhood system of MRF. MAP criterion is adopted to obtain segmentation results. We did a lot of contrast experiments, using this paper's algorithm, Markov Random Field algorithm in wavelet domain and Markov Random Field algorithm in Brushlet domain. Those experiment results indicate that this paper's algorithm is an effective segmentation algorithm for it can detect better texture direction information and keep better regional consistency than other two traditional algorithms.
\end{abstract}

Keywords- image segmentation; Overcomplete Brushlet transform; Gaussian Markov Random Field; Gibbs distribution; MAP criterion

\section{INTRODUCTION}

Texture image segmentation has always been one of difficult problems in the field of image segmentation, and its research goal is to explore a method which has the ability not only to effectively detect direction information but also to obtain consistent edges. Its core tasks include feature extraction and region segmentation ${ }^{[1]}$.

Segmentation methods based on Markov Random Field model ${ }^{[2]}$ are a class of statistical methods which have good theoretical bases and are good segmentation tools. In the process of image segmentation based on MRF, first of all to be done is to extract the feature parameters based on the random field model, so that the feature field of an image is constructed; then on the premise of the known feature field model, segmentation results are obtained by maximize the probability of the marker field. When the probability of the marker field is calculated, it is usually assumed that the probability of marker field has Markov property, namely the marker of each pixel is only affected by the markers of spatial adjacent pixels. Therefore, this model effectively considers the spatial topological relations between the pixels to ensure the consistency of segmented regions ${ }^{[3]}$.

The effectiveness of feature extraction is the key to improve the texture segmentation performance. In the past decades, popular methods in image segmentation based on MRF model were to combine wavelet transform with MRF [4-6]. Indeed, for some texture information, the segmentation effect in the wavelet domain is better than the one in the original pixel domain. However, when used to perform the segmentation of similar textures, wavelet has serious confusion. Because of limited directivity of wavelet, it is only suited to approximate point singulars of textures, it cannot approximate optimally and nonlinearly linear singular and surface singular functions. So wavelet is difficult to extract feature information of the complex texture image with different directions. In the process of feature extraction, it is required to reflect the anisotropy of the image texture.

With the deep research of the multiscale geometric analysis, Brushlet was developed by Meyer and Coifman for compression of highly texturized images ${ }^{[7]}$. For Brushlet's strong angle resolution, multilayer decomposition structure, and good properties to capture the direction of texture information, using it to extract image texture feature will receive better results. Elsa D. Angelini ${ }^{[8]}$ modified Brushlet to provide an overcomplete representation. Overcomplete multiscale representations are well suited for image analysis and enhancement, because they avoid aliasing effects introduced by critical sampling and yield a translation-invariant representation.

Based on above advantages, an effective segmentation algorithm is explored by applying the overcomplete Brushlet transform and MRF to the segmentation of texture images. While detecting the different directions, it also keeps the regional consistency. A number of experiments illustrate the effect of this method.

\section{BRUSHLET AND OVERCOMPLETE BRUSHLET}

Overcomplete Brushlet Transform is used to extract the feature of a texture image. Overcomplete Brushlet Transform is developed on the basis of Brushlet orthogonal base.

\section{A. Brushlet}

Brushlet base functions are orthogonal windowed Fourier bases. A smooth signal window function is divided into the adjacent interval, and then local Fourier analysis is performed in each interval, so as to construct the Brushlet bases.

The two partitions of $\mathbb{R}$ are defined as $\mathrm{U}_{j=-\infty}^{j=+\infty}\left[x_{j}, x_{j+1}\right]$ and $\mathrm{U}_{k=-\infty}^{k=+\infty}\left[y_{k}, y_{k+1}\right]$, and $h_{j}=x_{j+1}-x_{j}$ and $l_{k}=\mathrm{y}_{k+1}-\mathrm{y}_{k}$. Considering the tiling obtained by the lattice cubes $\left[x_{j}, x_{j+1}\right]$ 
$\otimes\left[y_{\mathrm{k}}, y_{\mathrm{k}+1}\right]$, a two-dimensional orthonormal Brushlet basis $w_{m, j}(x) \otimes \mathrm{w}_{n, k}(y)$ is expressed as follows,

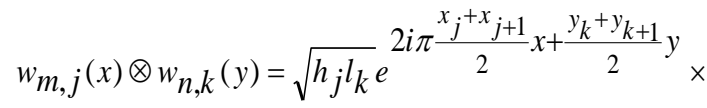

$$
\begin{aligned}
& \left\{(-1)^{m} \hat{b}_{\sigma}\left(h_{j} x-m\right)-2 i \sin \left(\pi h_{j} x\right) \hat{v}_{\sigma}\left(h_{j} x+m\right)\right\} \times \\
& \left\{(-1)^{n} \hat{b}_{\sigma}\left(l_{k} y-n\right)-2 i \sin \left(\pi l_{k} y\right) \hat{v}_{\sigma}\left(l_{k} y+n\right)\right\}
\end{aligned}
$$

where, $h_{j}$ and $l_{k}$ are scaling factors, $m$ and $n$ are translation factors, and $\hat{b}_{\sigma}$ is the window function of the Fourier transform supported on $[-1 / 2-\sigma, 1 / 2+\sigma] \otimes[-1 / 2-\sigma$, $-1 / 2+\sigma]$, and $\hat{v}_{\sigma}$ is the bump function supported on $[-\sigma,+\sigma]$ $\otimes[-\sigma,+\sigma]$.

The tensor product $w_{m, j}(x) \otimes w_{n, k}(y)$ is an oriented pattern oscillating with the frequency $\left(\left(x_{j}+x_{j+1}\right) / 2\right),\left(\left(\mathrm{y}_{k}+\right.\right.$ $\left.\left.\mathrm{y}_{k+1}\right) / 2\right)$ and localized at $\left(m / h j, n / l_{k}\right)$ with only one peak in frequency. The size of the pattern is inversely proportional to the size of the analyzing window.

Brushelet has a similar multilayer decomposition structure with wavelet packet. But each Brushelet base is a complex function with a phrase which represents an orientation of the Brushlet pattern. Considering the complexity of calculation, 2-layer Brushlet transform is used here. The sixteen sets of Brushlet with twelve different orientations is shown in Fig. 1. The orientations are associated with two different frequencies. The four lattice squares around the origin characterize the DC terms of the expansion. The other squares correspond to higher frequency textures. Because the coefficients are antisymmetric with respect to the origin, only the upper eight sub-bands need be extracted. Brushlet coefficients can be implemented efficiently by a folding technique and fast Fourier transform (FFT).

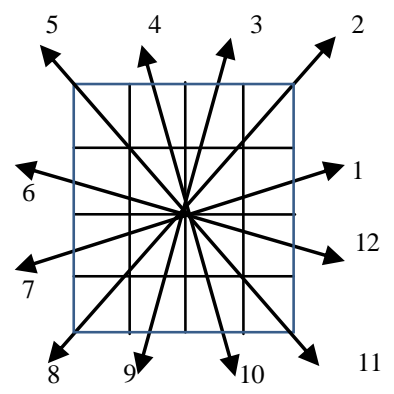

Figure 1. 12 orientations of 2-layer Brushlet decomposition

\section{B. Overcomplete Brushlet}

After every layer of Brushlet transform, the resolution of each subblock is reduced to a quarter of the original. Thus subblocks will be smaller and smaller. In order to ensure that the scope of each neighborhood is enough for the feature selection of each center point and aliasing effects introduced by critical sampling are avoided, a kind of overcomplete projection is used by padding the folded signals with zeros along each dimension before folding processing ${ }^{[8]}$. Each subinterval of the Fourier domain is projected onto a Brushstroke of dimension equal to the original number of elements. And then the size of each subblock equals to the size of the original image, thus the resolution of the local FT is increased. Therefore, there is a perfect homomorphism between the location of data points in the original image and the position of the coefficients in each projected subblock.

Just like Brushlet, the real part and the imaginary part of Overcomplet Brushlet have a lot of similarities, so only extracting the characteristics of upper 8 subblocks of the real component. The Overcomplete Brushlet transform can be implemented as follows:

- Compute 2-D FFT of the signal.

- $\quad$ Divide the upper half of the Fourier plane into 8 subblocks. A first expansion is performed with a portioning of the Fourier plane into four quadrants. And then each upper quadrant is further divided into four quadrants.

- Pad each subblock with zeros along each dimension.

- Fold the overlapping parts of the window $b_{\sigma}$ and of the hump function $v_{\sigma}$ back into the interval, across the endpoints of the interval, with some folding and unfolding operators.

- Calculate inside each subblock the 2-D FFT of the folded block and obtain the brushlet coefficients.

\section{IMAgE FEATURE FIELD MOdEL}

\section{A. Gaussian Markov Random Field Model}

Because Gaussian Markov Random Fields Model [9][10] considers both neighborhood information of a pixel within the same band and the relationship among the adjacent positions in the different bands, it's very suitable to construct the feature field model of a multispectral texture image. For single sample point $y_{s}$ in a data set $y=\left\{y_{1}, y_{2}, \ldots\right.$, $\left.y_{N}\right\}, y_{s}=\left\{y_{s}^{(1)}, y_{s}^{(2)}, \ldots, y_{\mathrm{s}}^{(D)}\right\}$ here $D$ is the number of bands of the sample point $y_{s}$, its conditional probability density function within the region $R$ is expressed as follows by the GMRF model ${ }^{[11]}$ :

$$
P\left(y_{s} \mid R\right)=\frac{\exp \left\{-\left(\left(e_{s}\right)^{T}(\Sigma)^{-1} e_{s}\right) / 2\right\}}{\left((2 \pi)^{D}|\Sigma|\right)^{1 / 2}}
$$

Where, $e_{s}=\left[e_{s}^{(1)}, e_{s}^{(2)}, \ldots, e_{s}^{(D)}\right]^{\mathrm{T}}$ is the gaussian noise vector with a zero mean, and the space correlation of pixels in the Multispectral image is expressed by

$$
e_{s}^{(j)}=\left(y_{s}^{(j)}-\mu^{(j)}\right)-\sum_{i=1}^{D} \sum_{r \in \eta_{S}} \theta_{j i}(r)\left(y_{S+r}^{(i)}-\mu^{(i)}\right)
$$

Where, $\mu=\left[\mu^{(1)}, \mu^{(2)}, \ldots, \mu^{(D)}\right]^{\mathrm{T}}$ is the mean vector for all pixels in the region $R . \eta_{s}$ is a collection of a series of offsets between the neighborhood of the position $s$ and $s$. Here the second order neighborhood of the position $s$ is used. And $\theta_{j i}$ is the neighborhood interaction coefficient of the jth band and the ith band in GMRF model. $\Sigma$ is the conditional covariance matrix, which is a symmetric matrix and is used to describe numerical characteristics of the relationship between any two bands ${ }^{[12]}$, and its mathematic formula is shown as follows:

$$
\Sigma=\left[\begin{array}{cccc}
v_{11} & v_{12} & \ldots & v_{1 D} \\
v_{21} & v_{22} & \ldots & v_{2 D} \\
: & : & : & : \\
v_{D 1} & v_{D 2} & \ldots & v_{D D}
\end{array}\right]
$$

Here, $v_{i j}$ is the mathematical expectation of $e^{(i)} e^{(j)}$. 


\section{B. Parameter Estimation}

The technique used to estimate the parameters of GMRF model here is the least squares estimation, which is proposed to in [13]. Its parameter estimation formula is

$$
\begin{aligned}
& \theta=\left[\sum_{s \in R} q_{s} q_{s}^{T}\right]^{-1}\left(q_{s} \sum_{s \in R} y_{s}^{T}\right) \quad \text { and } \\
& \Sigma=\frac{1}{N} \sum_{s \in R}\left(y_{s}-\theta^{T} q_{s}\right)\left(y_{s}-\theta^{T} q_{s}\right)^{T}
\end{aligned}
$$

Here, $q_{s}=\operatorname{col}\left[y_{s+r}-y_{s-r}\right]$.

\section{MARKER FIELD MODEL}

Let the set of marker field of an image be $x$, $x=\left\{x_{s} \mid s \in S\right\}$, where $S$ is the collection of pixel locations, and let the value set of class marker $x_{s}$ be $\{1,2, \cdots, K\}$, where $K$ is the number of the image classification. Here the second order neighborhood system of MRF is used to describe the image marker field model. According to Hammersley-Clifford theorem, the prior probability $P(x)$ is obeyed to Gibbs distribution ${ }^{[14]}$.

$$
P(x)=Z^{-1} \times e^{-U(x)}
$$

Here, $U(x)=\sum_{c \in C} V_{c}(x)$ is the energy function and the sum of potential functions of all perssads in the perssad collection $C$, and $Z=\sum_{x \in S} e^{-U(x)}$ is the normalized constant. In this paper, the potential function $V_{c}$ is gotten by a multilevel logic model, which is abbreviated by MLL, and $V_{c}$ is here defined as:

$$
V_{c}\left(x_{i}, x_{j}\right)=\left\{\begin{array}{lc}
1 & \left(x_{i}=x_{j}\right) \\
-1 & \left(x_{i} \neq x_{j}\right)
\end{array}\right.
$$

Therefor, the local probability of MLL model is

$$
\mathrm{P}\left(x_{i} \mid x_{N_{i}}\right)=\exp \left[-n_{i}\left(x_{i}\right)\right] / \sum_{x_{i} \in L} \exp \left[-n_{i}\left(x_{i}\right)\right]
$$

Where, $n_{i}\left(x_{i}\right)$ is the number of markers which are not equal to $x_{i}$ in the neighborhood of position $i$.

\section{SEGMENTATION ALGORITHM}

\section{A. Segmentation Criteria}

The maximum a posteriori probability criterion $^{[15]}$ based on Bayesian theory is one of the most optimal segmentation criterions, and it is usually used in the image segmentation based on MRF. The MAP criterion obtains the image segmentation results through maximizing the marker field probability on the premise of the known feature field. It is expressed by

$$
\hat{x}=\arg \max P(x \mid y)=\arg \max P(y \mid x) P(x)
$$

Let $E, E f$ and $E l$ represent respectively the total energy, the energy of the feature field and the energy of the marker field. $E f=-\sum \log P(y \mid x)$ and $E l=-\sum \log P(x)$. And then the total energy is as follows:

$$
E=E f+E l
$$

Therefore, MAP criterion can be expressed by energy function as follows:

$$
\hat{x}=\arg \min E=\arg \min (E f+E l)
$$

\section{B. Segment Process}

The image segmentation process is as follows:
- Set the number of image classification and the number of iterations.Compute 2-D FFT of the signal.

- Extract image texture feature. Two layer Overcomplete Brushlet transform is carried out for the original image, thus the characteristic matrix of upper eight sub-bands of the real part are gotten.

- The least squares parameter estimation is used to estimate the parameters of GMRF model, as equation (5) and (6) are shown, while the coefficient matrix got in (b) are looked as a reality of the feature field.

- The feature field energy $E f$ of each position is calculated according to equation (2) - (4).

- Calculate the marker field Energy $E l$ of each position by equation (9).

- After the energy component of each pixel using the equation(11) is calculated, the classification mark corresponding with minimum energy is looked as the new marker of this pixel position.

- Repeat the above last four steps until the end condition is satisfied.

So far the markers of pixels gotten at last are segment results.

\section{EXPERIMENTAL RESULTS AND ANALYSIS}

In matlab7.0, three algorithms including Markov Random Field algorithm in wavelet domain (WMRF algorithm), Markov Random Field algorithm in Brushlet domain (BMRF algorithm), and Gauss-markov Random Field algorithm in overcompete Brushlet domain (OBMRF algorithm) proposed in this paper are respectively used to carry on the contrast experiments for a variety of synthetic texture images. Fig.2, Fig.3 and Fig.4 show the results of segmentation experiments for two types of images, and TABLE I shows evaluations of segmentation quality of each image corresponding of three algorithms, including the overall classification accuracy and Kappa coefficient.

By Comparing images in fig. 2 with those in fig.3, when image 1 shown in the fig.2(a) is looked as two classes of textures, the segmentation result gotten by WMRF algorithm is very good, but when it is looked as include three classes of textures, WMRF algorithm cannot distinguish between texture 1 and 2 , as shown in fig. 3 (b) .This indicates that the wavelet is only able to identify point singularities in an image, but the recognition ability for line singularities and curve singularities of texture information is very poor.

As shown in fig.4, there are four classes of textures in image2. When image 2 is segmented with WMRF algorithm, the very serious confusion appears; when it is segmented with BMRF algorithm, the segmentation effect has a degree of improvement; however, the effect has obvious improvement, when OBMRF is used.

These experimental results show that the segment method proposed in this paper is better than traditional wavelet transformation method in these respects of the segmentation error rate, region consistency, and edge accuracy. And to a certain extent, they also show that overcomplete Brushlet transformation used as the domain of the feature field can obtain better segmentation effect than Brushlet, when markov random field is used to segment an image. 


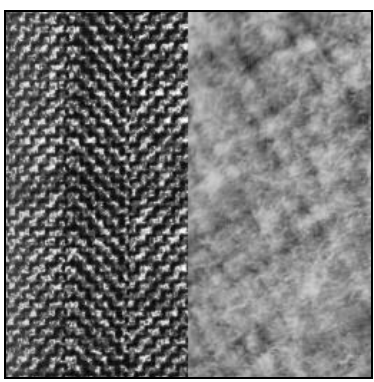

(a)

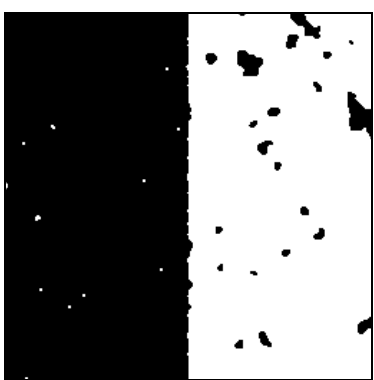

(b)

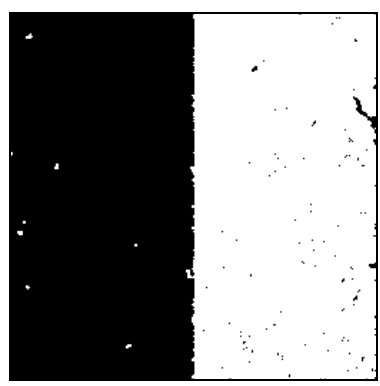

(c)

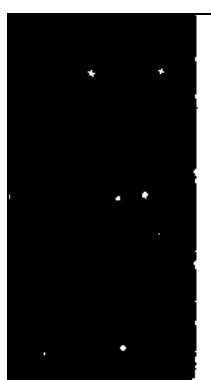

(d)

Figure 2. (a) the original synthetic image1. (b), (c) and (d) are respectively its segmentation results gotten by WMRF, BMRF and OBMRF algorithm when image1 is looked as the image with two classes of textures.

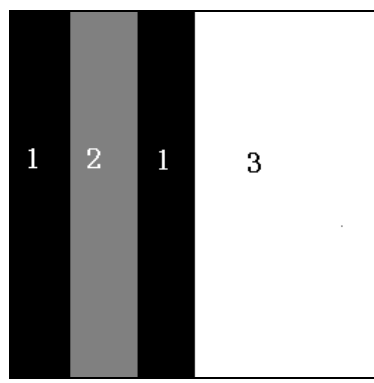

(a)

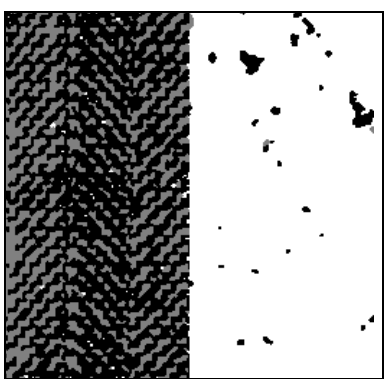

(b)

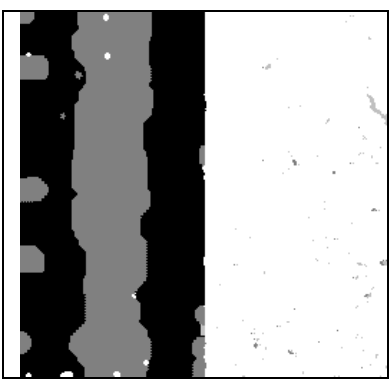

(c)

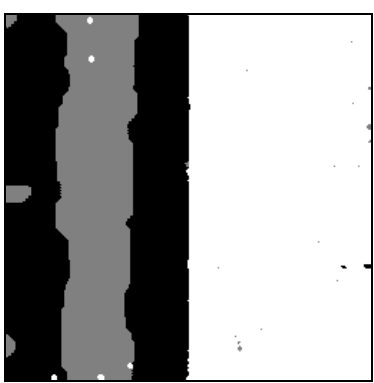

(d)

Figure 3. (a) the segmentation template of image1. (b), (c) and (d) are respectively its segmentation results gotten by WMRF, BMRF and OBMRF algorithm when image 1 is looked as the image with three classes of textures.

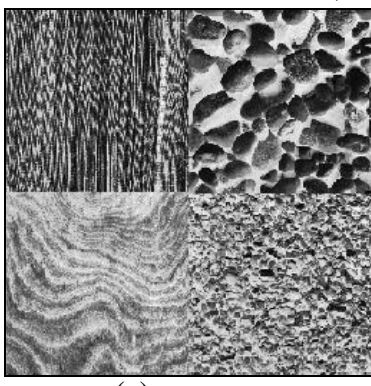

(a)

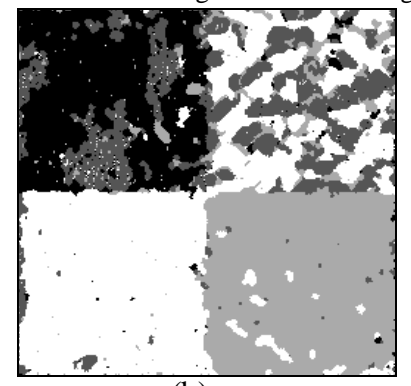

(b)

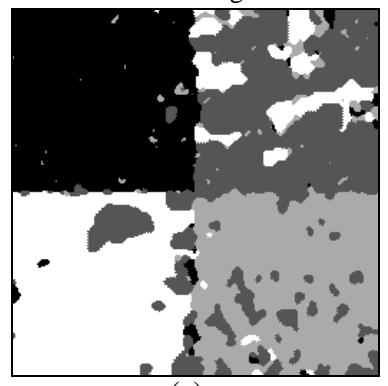

(c)

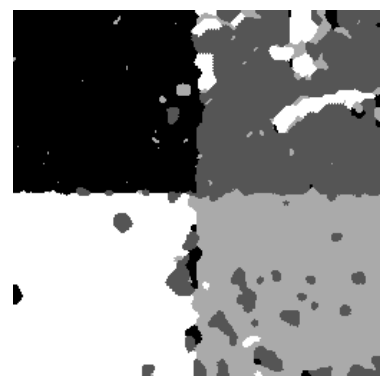

(d)

Figure 4. (a) the original synthetic image 2. (b), (c) and (d) are respectively its segmentation results gotten by WMRF, BMRF and OBMRF algorithm .

TABLE I Performance Comparison

\begin{tabular}{|c|c|c|c|c|}
\hline \multicolumn{2}{|c|}{$\begin{array}{c}\text { Segmentation } \\
\text { Quality }\end{array}$} & \multicolumn{3}{c|}{ Segmented Images } \\
\cline { 3 - 5 } & $\begin{array}{c}\text { Image 1 } \\
\text { with two } \\
\text { classes of } \\
\text { textures }\end{array}$ & $\begin{array}{c}\text { Image 1 } \\
\text { with three } \\
\text { classes of } \\
\text { textures }\end{array}$ & Image 2 \\
\hline \multirow{4}{*}{ WMRF } & $\begin{array}{c}\text { Kappa } \\
\text { coefficient }\end{array}$ & 0.9471 & 0.5954 & 0.4025 \\
\cline { 2 - 5 } & $\begin{array}{c}\text { overall } \\
\text { classification } \\
\text { accuracy }\end{array}$ & 0.9736 & 0.7532 & 0.5031 \\
\hline \multirow{3}{*}{ BMRF } & $\begin{array}{c}\text { Kappa } \\
\text { coefficient }\end{array}$ & 0.9770 & 0.9141 & 0.5577 \\
\cline { 2 - 5 } & $\begin{array}{c}\text { overall } \\
\text { classification } \\
\text { accuracy }\end{array}$ & 0.9885 & 0.9464 & 0.6373 \\
\hline \multirow{2}{*}{ OBMRF } & $\begin{array}{c}\text { Kappa } \\
\text { coefficient }\end{array}$ & 0.9835 & 0.9534 & 0.6283 \\
\cline { 2 - 5 } & $\begin{array}{c}\text { overall } \\
\text { classification } \\
\text { accuracy }\end{array}$ & 0.9931 & 0.9740 & 0.6945 \\
\hline
\end{tabular}

\section{CONCLUSION}

A combination segmentation method of texture images based on Overcomplete Brushlet and Gauss-MRF in this paper is proposed. The overcomplete Brushlet as a multiscale geometric analysis tool can effectively describe the texture feature, while the MRF model promotes the region consistency and accurate edges, therefore, a combination of both makes sure that good segmentation results are achieved. The experimental results also show that the algorithm proposed in this paper is effective.

\section{ACKNOWLEDGMENT}

the research in this paper was supported by the Project of Education Department of Liaoning Province of China(Project No.L2013422), and the Project of Liaoning Provincial Federation Social Science Circles of China (Project No. 2014lslktzdian-04).

\section{REFERENCES}

[1] Fumiaki T, Saburo T. Computer Analysis of Visual Textures [M]. Boston: Kluwer Academic Publishers, 1990. 
[2]Stan ZQ.Li. Markov Random Field Modeling in Image Analysis [M]. NewYork: Springer-Verlag, 2009.

[3] Chathurika D W ,Sasan M, et al. Gaussian Markov Random Field Based Improved Texture Descriptor for Image Segmentation [J]. Image and Vision Computing,2014,11(32): 884-895.

[4]Noda H, Shirazi M N, Kaw aguchi E. MRF-Based Texture Segmentation Using Wavelet Decomposed Images[J]. Pattern Recognition, 2002, 35:771-782.

[5] Varun G, Loveleen K, Uma K. Color Satellite Image Segmentation Using Markov Random Field and Multiresolutional Wavelet Transform[J]. International Journal of Computer Applications in Engineering Sciences (IJCAES),2011,1(1):75-79.

[6] Emmanouil A, Dionisis C, et al. A wavelet-based Markov random field segmentation model in segmenting microarray experiments[J]. Computer Methods and Programs in Biomedicine, 2011,104(3):307-315.

[7] Meyer F G, Coifman R R. Brushlets: A Tool for Directions Image Analysis and Image Compression[J]. Applied and Computational Harmonic Analysis, 1997, 6(4):147-187.

[8] Elsa D A, Andrew L, et al. LV Volume Quantification via Spatiotemporal Analysis of Real-Time 3-D Echocardiography[J].
IEEE TRANSACTIONS ON MEDICAL IMAGING, 2001, 20(6):457-469.

[9]Havard R, Leonhard H. Gaussian Markov Random Fields Theory and Applications[M]. London: Chapman \& Hall/CRC, 2005.

[10]Mridula J, Dipti P.Unsupervised Segmentation of Multispectral Textured Images using GA-GMRF model[J]. International Journal of Computer Science \& Emerging Technologies,2010,1(4): 230-235.

[11] Zhao Yindi, Zhang Liangpei, et al. Universal Markov Random Fields and Its Application in Multispectral Textured Image Classification[J]. Journal of Remote Sensing, 2006,10(1):123-130.

[12] Tang G A, Zhang Y S, et al. Remote Sensing Digital Image Processing[ M ]. Peking:Science Press, 2004.

[13]Wang L, Liu J. Texture Classification Using Multiresolution Markov Random Field Models[J]. Patter Recognition Letters, 1999, 20:171-182.

[14]Chan M, Levitan E, et al. Image-modeling Gibbs distributions for Bayesian restoration[A]. Proceedings of the IEEE Southwest Symposium, 1994, 1:21-24.

[15] Butler C S, Miller M I. Maximum a posteriori estimation for SPECT using regularization techniques on massively parallel computers[J]. IEEE transactions on medical imaging, 1993,12: 84-9. 